\title{
Studies in the Maloideae (Rosaceae) 1- Chaenomeles Lindley and Cydonia Miller
}

\author{
Mohammed H. Loutfy \\ Ali A. A. El-Mashad
}

And

Ehab A. Kamel

Department of Biological Sciences and Geology, Faculty of Education, Ain Shams University, Roxy, Cairo, 11341, Egypt.

\begin{abstract}
Loutfy M. H. A, El-Mashad A. A. A. \& Kamel E. A. Studies in the Maloideae (Rosaceae) I- Chaenomeles Lindley and Cydonia Miller. Taeckholmia 19(2):97-114.

Macromorphological characters, SEM of seed coat surface criteria, seed coat anatomy and seed protein electrophoresis aspects, were used to re-assess the taxonomic relationships between the genera Chaenomeles and Cydonia. Characters were analyzed by the NTsys-pc. program package, using the UPGMA clustering method. The dendrograms produced were discused and showed a close relationship between Chaenomeles sinensis Koehne and Cydonia oblonga Miller. The result gives support to the merging of the former taxon in the genus Cydonia as presented in Mabberley (1997).
\end{abstract}

Key words: Chaenomeles - Cydonia - Electrophoresis - Maloideae - Rosaceae - Systematics

\section{Introduction}

The Maloideae (Pyroideae) is a natural sub-family of the Rosaceae, with 23-28 genera and 940-1110 species (Phipps et al., 1990 and Robertson et al., 1991). It stands apart from all the rest of the family by several aspects as :- Basic chromosome numbers of 17 (except in some American species of Crataegus), unique fruit structure (pome), narrow medullary rays and a well developed seed testa (Stebbins 1950, Mclean \& Cook 1956, Corner 1976 and Heywood 1993). As it is the case with most natural groups, the differences between the genera of the Maloideae are slight, generic limits cannot be drawn sharply between many of the apparent genera (Fernald 1947 and Bailey 1949 b). Inconsistency of the main generic characters has generated a great deal of disagreement in the taxonomic treatment of the group (Aldasoro et al., 1998). The genera Chaenomeles and Cydonia (Tribe Sorbeae) are closely related, being differentiated from each other by few morphological and floral characters (Bailey 1949 a, Eames 1961 and Mabberley 1997). The two genera hybridize well with each other and between their species and numerous cultivars are now available (Hillier 1981 and Beckett 1983). Bailey \& Bailey (1976) and Mabberley (1997) stated that Chaenomeles contains (3-4) species: C. cathayensis (Hemsl) Schneid., C. japonica (Thumb). Lindl. and C. speciosa (Sweet) Nakai, while Cydonia contains two species: $C$. oblonga Mill. and $C$. sinensis Thouin. However, controversies still exist, as to the delimitation of the two genera or at the sub-species level.

Received 12 May 1999. Revision accepted 27 October 1999. 
Several studies were made on the two genera and the subfamily Maloideae using different morphological and molecular criteria. Weber (1964) gave a detailed account on the genus Chaenomeles and its relations with other genera of the Maloideae. He reported that Cydonia, Malus and Pyrus could hybridize among themselves. Sterling (1966) and Kalkman (1988) proposed that Pyrus may have branched from the ancestor of Cydonia before the latter acquired the pluriovulate carpels. Phipps et al., (1990) proposed a checklist of the genera of the Maloideae. According to them, the genus Chaenomeles contains 4 species. Phipps et al., (1991) and Robertson et al., (1991) discussed the phylogeny of the Maloideae and presented a synopsis of genera in the sub-family in an attempt to aid in their delimitation. Rohrer et al., (1991) showed that the fruit morphology supports a close relationship between the Chaenomeles species. Robertson et al., (1992) stated that Pseudocydonia (Cydonia sinensis) differed from all the other genera of the Maloideae, in possessing leaves with cylindrical gland tipped teeth. Rohrer et al., (1994) stated that Cydonia and Pyrus are sister groups according to a cladistic analysis utilizing some morphological aspects. The same result was arrived at by Campbell et al., (1995) utilizing molecular criteria and Aldasoro et al., (1998) by studying the pome anatomy.

The significance of seed structure in taxonomic and phylogenetic studies has been emphasized by many authors (Netolitzky 1926, Martin 1946, Duke 1961, Corner 1976 \& 1992, Rezk 1980 \& 1987). SEM of seed coat surface is useful in the identification and classification of various taxa (Stant 1973, Brisson \& Peterson 1976, Barthlott 1981 and Boesewinkel \& Bouman 1984). A comparison of surface scan patterns of the seed coat has efficiently been used in studying species of some genera including Vigna (Kumar et al., 1984), the Abutileae (Khushk \& Vaughan 1986), the Vicieae (Chernoff et al., 1992) and Ranunculus (Xuhan \& Van-Lammeren, 1994). As far as the literature cited, no attempt has been made for studying the seeds of Chaenomeles \& Cydonia in particular, except Corner (1976) who studied the seed coat anatomy of Chaenomeles japonica Lindl. \& Cydonia oblonga Mill. According to him, the former species differs from the latter in that the testa is scarcely mucilaginous and the cells scarcely radially elongate. Rudenko \& Rotaru (1988) studied the variation in seed anatomy, especially the epidermis and testa structures in diploid varieties of Cydonia oblonga and Chaenomeles japonica $(2 n=34)$, allotetraploid $\mathrm{F}_{2}$ Cydonia and Malus hybrids $(2 n=68)$ and Pyrus and Cydonia hybrids $(2 n=34)$. Differences were found between the species and their hybrids. As to the Maloideae in general, one would refer to the works of Pechoutre (1902), Netolitzky (1926) and Sterling (1966).

On the other hand, seed proteins are highly stable, being unaffected by environmental conditions Haborne \& Turner (1984). Thus electrophoretic patterns of total seed protein (protein profiles) as revealed by polyacrylamide gel electrophoresis in the presence of sodium dodecyl sulfate (SDS-PAGE) have provided a valid source of taxonomic evidence and were used to address taxonomic relationships at the generic and specific levels, for example Vigna (Paino et al., 1993), Phaseolus (Schmit et al., 1996) Sesbania (Badr et al., 1998) \& Nigella (Jensen 1984).

The present study aims at using seed characters (macro and micromorphological) including seed coat anatomy and SEM of seed coat surface, together with characters from vegetative morphology, seed storage protein profiles and numerical taxonomic methods to help in clarifying and delimiting the two genera studied. 


\section{Materials and Methods}

Seeds of the examined species and their sources are listed in Table (1). Macromorphological aspects were collected from relevant literature (Makins 1948, Bailey 1949 a, Bean 1950, Bailey \& Bailey 1976, Hillier 1981, Beckett 1983 and Mabberley 1997).

Table (1): Sources of the studied Taxa.

\begin{tabular}{|c|c|c|c|c|}
\hline Taxon & $\begin{array}{l}\text { English } \\
\text { name }\end{array}$ & Source & $\begin{array}{l}\text { Country } \\
\text { of origin }\end{array}$ & $\begin{array}{l}\text { Distribution } \\
\text { in Egypt }\end{array}$ \\
\hline $\begin{array}{l}\text { 1) Chaenomeles japonica (Lindl.) Spach } \\
\text { syn: Chaenomeles Maulei Schneid. } \\
\text { syn: Cydonia Maulei Moore } \\
\text { syn: Pyrus japonica Thunb. }\end{array}$ & $\begin{array}{c}\text { Dwarf } \\
\text { Japanese } \\
\text { quince }\end{array}$ & TMPRS & Japan & 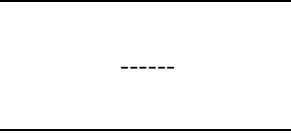 \\
\hline $\begin{array}{l}\text { 2) Chaenomeles sinensis Koehne. } \\
\text { syn: Cydonia sinensis Thouin } \\
\text { syn: Pseudocydonia sinensis Schneid. }\end{array}$ & $\begin{array}{l}\text { Chinese } \\
\text { quince }\end{array}$ & TMPRS & China & $\begin{array}{l}\text { Rare, some specimens } \\
\text { were planted in Bircher's } \\
\text { Garden at El Saff }\end{array}$ \\
\hline $\begin{array}{l}\text { 3) Chaenomeles speciosa Nakai. } \\
\text { syn: Chaenomeles lagenaria Koidz. } \\
\text { syn: Cydonia lagenaria Loisel } \\
\text { syn: Chaenomeles japonica Hort. } \\
\text { syn: Cydonia japonica Pers. } \\
\text { syn: Pyrus japonica } \text { Sims. }\end{array}$ & $\begin{array}{l}\text { Japanese } \\
\text { quince }\end{array}$ & TMPRS & $\begin{array}{l}\text { China } \\
\& \\
\text { Japan }\end{array}$ & $\begin{array}{l}\text { Rare, some specimens } \\
\text { were planted in Bircher's } \\
\text { Garden at El Saff } \\
\text { (Bircher 1960) }\end{array}$ \\
\hline $\begin{array}{l}\text { 4) Cydonia oblonga Mill. } \\
\text { syn: Cydonia vulgaris Pers. } \\
\text { syn: Pyrus cydonia L. }\end{array}$ & $\begin{array}{l}\text { Edible } \\
\text { quince }\end{array}$ & OBS & $\begin{array}{l}\text { Unknown, } \\
\text { cultivated } \\
\& \\
\text { naturalized } \\
\text { in many } \\
\text { places of } \\
\text { the Old } \\
\text { World } \\
\end{array}$ & $\begin{array}{l}\text { Cultivated on a small } \\
\text { scale for its edible fruits. } \\
\text { It is also used as a stock } \\
\text { for grafting pears and } \\
\text { apples. (Bircher 1960) }\end{array}$ \\
\hline
\end{tabular}

For study of seed coat surface using SEM, two seeds were mounted with colloidal silver on copper stubs and coated with a thin layer of gold in Polaron E 5000. The epidermal seed coat was photographed by a JEOL- Scanning Microscope at the central lab. of Faculty of Science - Alexandria University. The terminology of Stearn (1966), Barthlott (1981) and Boesewinkel \& Bouman (1984) has been used to describe the characteristics of the seed coat.

In addition, transverse sections were made in the seed coats of the studied taxa by hand microtome at $15-20 \mu$ at the Faculty of Science - Ain shams University. Sections were photographed using Carl-Zeiss photomicroscope III at a magnification of $\mathrm{x}=200,256$ $\& 320$ at the Faculty of Education - Ain shams University. Description and terminology presented by Corner (1976) has been used to describe the anatomical features of the seed coat.

For SDS-PAGE electrophoresis, three replicates of $0.1 \mathrm{gm}$ of seeds were mixed, each with an equal weight of pure, clean, sterile fine sand and powdered using mortar and pestle. Extraction of proteins was carried out using four buffers; Tris-Glycine ( $\mathrm{pH} 8.2$ ), Tris-EDTA ( $\mathrm{pH} \mathrm{8.8),} \mathrm{Tris-} \mathrm{HCl}$ (8.0) in the presence of 2-mercaptoethanol (under reducing 
condition) and Tris- $\mathrm{HCl}$ ( $\mathrm{pH} 8.0$ ) without $2 \mathrm{ME}$ (under non-reducing condition). The powder was homogenized with $1 \mathrm{ml}$ of each buffer for $2 \mathrm{hr}$. at $20{ }^{\circ} \mathrm{C}$. SDSpolyacrylamide gel electrophoresis was carried out in $12.5 \%$ acrylamide gels in TrisGlycine running buffer ( $\mathrm{pH} 8.3$ ) at $150 \mathrm{~V}$ for $3 \mathrm{hr}$. using a low molecular weight protein of Sigma as a marker in each run. Gels were then stained in Comassie brilliant blue R-250 for $30 \mathrm{~min}$., destained, photographed and molecular weight values for subunits were determined by comparison with standard proteins as described by Matta et al. (1981). Analysis was carried out using pro-analyzer version 2.0.

For the data analysis, the total number of the recorded characters (178) in each taxon, were scored, combined together in four sets of data \& coded for creating the data matrix of computation:

a) Morphological characters of whole plant.

b) Anatomical characters of seed coat (LM).

c) Morphological characters of seed coat (SEM).

d) SDS-PAGE characters.

e) All characters combined.

The presence or absence of each 178 different characters was treated as a binary character in a data matrix i.e. coded 1 and 0 respectively (Table 2).

The relationships between the taxa studied, expressed by average taxonomic distance (dissimilarity), have been demonstrated as phenograms, based on the analysis of the recorded characters using the NTsys program package for IBM-pc as described by Rohlf (1989).

\section{Results}

I. Selected macromorphological features of the studied taxa (After Makins 1948, Bailey 1949 a, Bean 1950, Bailey \& Bailey 1976, Hillier 1981, Beckett 1983 and Mabberley 1997) are summarized below:

\section{(1). Chaenomeles japonica (Thumb.) Lindl.}

A thorny semi-deciduous sub-shrub $90 \mathrm{~cm}$ high. Leaves simple, leaf base with large stipules, blade margin serrate to crenate and apex accuminate to obtuse. Young branchlets and leaves downy. Flowers $3 \mathrm{~cm}$ wide, orange red to scarlet, in clusters on previous year's wood, styles joined at base. Fruit $3 \mathrm{~cm}$ wide and yellowish red. Seeds globose to pear shaped and dark brown.

\section{(2). Chaenomeles sinensis Koehne.}

(=Cydonia sinensis Thouin., Pseudocydonia sinensis Schneider)

Spineless semi deciduous shrub 3-6 m high. Leaves simple. Leaf base with small stipules. Petiole short and pubescent with glandular teeth. Blade elliptical to obovate. Margin serrate. Apex acuminate. Flowers solitary, pale pink, sepals reflexed and serrulate, styles joined at base. Fruit $15 \mathrm{~cm}$ wide, oblong, yellow and fragrant. Seed pear shaped and dark brown. 


\section{(3). Chaenomeles speciosa (Sweet) Nakai}

Thorny shrub $3 \mathrm{~m}$ high. Semi deciduous. Leaves glabrous and simple $3-4 \mathrm{~cm}$. Leaf base with large stipules. Blade oblong to ovate. Margin serrate and apex acuminate. Flowers in small clusters, scarlet red, styles joined at base. Fruit globose to ovoid, medium sized and green yellow. Seeds pear shaped and dark brown.

\section{(4). Cydonia oblonga Miller}

Spineless shrub 4-6 m high. Deciduous. Leaves simple with small stipules, petiole short and downy. Blade ovate, margin entire and apex acute. Flowers solitary, white to pale pink. Styles free . Fruit pyriform large and fragrant. Calyx persistent. Seeds pear shaped and slightly curved.

II. Anatomical aspects of the examined seed coat by LM (Terminology after Corner 1976) are summarized as follows:

(1). Chaenomeles japonica (Thunb.) Lindl.

Testa: Outer epidermal cells illdefined. Mesophyll: Several layers of highly lignified cells. The outer ones narrow and irregularly rectangular and the inner ones irregular hexagonal.

Tegmen: 3-4 layers of cells, outer ones crushed, inner ones narrow rectangular with a dark brown pigmentation.

Endosperm: Two layers thick.

\section{(2). Chaenomeles sinensis Koehne.}

(= Cydonia sinensis Thouin., Pseudocydonia sinensis Schneider)

Testa: Outer epidermal cells illdefined, some possess long pointed hairs. Mesophyll: Several layers of irregularly hexagonal highly lignified cells, the inner ones crushed.

Tegmen: 2-3 layers of narrow irregular rectangular cells with brown pigmentation.

Endosperm: 4-6 layers thick.

\section{(3). Chaenomeles speciosa (Sweet) Nakai.}

Testa: Outer epidermal cells illdefined. Mesophyll: Several layers of highly lignified irregular cells.

Tegmen: Illdefined.

Endosperm: 4-5 layers thick, some vacuoles or air lacunae are seen.

\section{(4). Cydonia oblonga Miller.}

Testa: Outer epidermal cells illdefined. Many possess very long narrow pointed hairs. Mesophyll: Composed of several layers of highly lignified irregular cells, the inner ones crushed. 
Tegmen: Outer layers crushed, the inner layers irregularly rectangular with dark pigmentation.

Endosperm: 8-10 layers.

III. Description and terminology of examined seed coat by SEM (after Stearn 1966, Barthlott 1981 and Boesewinkel \& Bouman 1984) are summerized as follows:

(1). Chaenomeles japonica (Thunb.) Lindl.

Spermoderm reticulate to colliculate. Epidermal cells monomorphic, isodiametric and irregularly pentagonal to hexagonal in shape. Anticlinal walls straight, thin, slightly raised and highly striated. Striations occasionally radiating, forming short ridges. Periclinal walls slightly convex, generally smooth with few striations.

\section{(2). Chaenomeles sinensis Koehne. \\ (Cydonia sinensis Thouin., Pseudocydonia sinensis Schneider)}

Spermoderm reticulate to colliculate. Epidermal cells rounded and monomorphic. Anticlinal walls curved, very thin, flat and striated. Periclinal walls flat to slightly convex and highly striated.

\section{(3). Chaenomeles speciosa (Sweet) Nakai.}

Spermoderm reticulate. Epidermal cells monomorphic, pentagonal to round. Anticlinal walls curved, very thick and highly raised. Periclinal walls flat to slightly concave, rough and slightly tuberculate.

\section{(4). Cydonia oblonga Miller.}

Spermoderm irregularly reticulate. Epidermal cells monomorphic and irregularly hexagonal. Anticlinal walls wavy, slightly thick, slightly raised and highly striated. Periclinal walls flat and highly striated. Long narrow hair-like structure or protrusions appear all over the cell wall.

IV. The electrophoretic banding patterns of the different buffers extracted proteins are shown in Fig. 3 (a-d). The distribution of protein bands in the different taxa based on their molecular weight is shown in Table (2). Chaenomeles japonica was found to have the highest number of band (20), whereas the lowest number (12) was found in Cydonia oblonga. While the highest molecular weight protein (103 KD) was found in Chaenomeles speciosa, the lowest molecular weight ( $3 \mathrm{KD})$ was found in Chaenomeles speciosa and Cydonia oblonga.

V. The numerical analysis of the recorded characters are summarized as follow;

The phenogram produced by cluster analysis based on 89 morphological characters clearly divided the four species into two groups (Chaenomeles japonica \& Chaenomeles speciosa) and (Chaenomeles sinensis \& Cydonia oblonga) at 1.47 
level. The first two species are separated at 1.30 average taxonomic distance and the other two species are separated at 1.31 average taxonomic distance (Fig. 4-a).

Table (2): Characters used in the numerical analysis and their codes.

$0=$ absent $; 1=$ present $; \mathrm{A}=$ Chaenomeles japonica $; \mathrm{B}=$ Chaenomeles sinensis $;$

$\mathrm{C}=$ Chaenomeles specios $; \mathrm{D}=$ Cydonia oblonga

\begin{tabular}{|c|c|c|c|c|c|c|c|c|}
\hline \multirow{2}{*}{ No } & \multicolumn{8}{|c|}{ I- Morphological characters: } \\
\hline & \multicolumn{4}{|c|}{ Character } & $\mathrm{A}$ & B & $\mathrm{C}$ & $\mathrm{D}$ \\
\hline 1 & \multirow{6}{*}{\multicolumn{3}{|c|}{ Habit }} & Sub-shrub. & 1 & 0 & 0 & 0 \\
\hline 2 & & & & Shrub. & 0 & 1 & 1 & 1 \\
\hline 3 & & & & $90 \mathrm{~cm}$. & 1 & 0 & 0 & 0 \\
\hline 4 & & & & $3-6 \mathrm{~m}$. & 0 & 1 & 0 & 0 \\
\hline 5 & & & & $3 \mathrm{~m}$. & 0 & 0 & 1 & 0 \\
\hline 6 & & & & $4.5-6 \mathrm{~m}$. & 0 & 0 & 0 & 1 \\
\hline 7 & \multirow{7}{*}{ 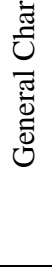 } & \multirow{2}{*}{\multicolumn{2}{|c|}{ Texture }} & \begin{tabular}{|l} 
Thomy. \\
\end{tabular} & 1 & 0 & 1 & 0 \\
\hline 8 & & & & Spineless. & 0 & 1 & 0 & 1 \\
\hline 9 & & \multirow{2}{*}{\multicolumn{2}{|c|}{ Leaf duration }} & Deciduous. & 0 & 0 & 0 & 1 \\
\hline 10 & & & & Semi-deciduous. & 1 & 1 & 1 & 0 \\
\hline 11 & & \multirow{3}{*}{\multicolumn{2}{|c|}{ Origin }} & China. & 0 & 1 & 1 & 0 \\
\hline 12 & & & & Japan. & 1 & 0 & 0 & 0 \\
\hline 13 & & & & Iran to Turkistan. & 0 & 0 & 0 & 1 \\
\hline 14 & \multirow{15}{*}{ 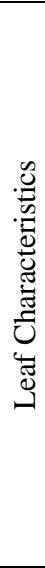 } & \multirow{2}{*}{$\begin{array}{l}\text { Leaf } \\
\text { base }\end{array}$} & \multirow[t]{2}{*}{ Stipules } & Very large. & 1 & 0 & 1 & 0 \\
\hline 15 & & & & Small. & 0 & 1 & 0 & 1 \\
\hline 16 & & \multirow{5}{*}{ Petiole } & Gland & Present or absent & 0 & 1 & 0 & 0 \\
\hline 17 & & & \multirow[t]{2}{*}{ Length } & Short. & 0 & 1 & 0 & 1 \\
\hline 18 & & & & \begin{tabular}{|l} 
Varying. \\
\end{tabular} & 1 & 0 & 1 & 0 \\
\hline 19 & & & \multirow[t]{2}{*}{ Texture } & Pubescent. & 1 & 1 & 0 & 1 \\
\hline 20 & & & & Glabrous. & 0 & 0 & 1 & 0 \\
\hline 21 & & \multirow{5}{*}{$\begin{array}{l}\text { Leaf } \\
\text { blade }\end{array}$} & Type & Simple. & 1 & 1 & 1 & 1 \\
\hline 22 & & & \multirow{4}{*}{ Shape } & Oblong to ovate. & 0 & 0 & 1 & 0 \\
\hline 23 & & & & Ovate. & 0 & 0 & 0 & 1 \\
\hline 24 & & & & Ovate to obovate. & 1 & 0 & 0 & 0 \\
\hline 25 & & & & \begin{tabular}{|l|} 
Ellipt. to obovate. \\
\end{tabular} & 0 & 1 & 0 & 0 \\
\hline 26 & & \multirow{3}{*}{\multicolumn{2}{|c|}{ Leaf Margin }} & Entire. & 0 & 0 & 0 & 1 \\
\hline 27 & & & & Crenate to serrate & 1 & 0 & 0 & 0 \\
\hline 28 & & & & Sharp serrate. & 0 & 1 & 1 & 0 \\
\hline 29 & \multirow{9}{*}{ 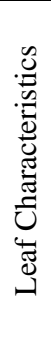 } & \multirow{3}{*}{\multicolumn{2}{|c|}{ Leaf Apex }} & Acute to obtuse. & 1 & 0 & 0 & 0 \\
\hline 30 & & & & Acuminate. & 0 & 1 & 1 & 0 \\
\hline 31 & & & & Acute. & 0 & 0 & 0 & 1 \\
\hline 32 & & \multirow{2}{*}{\multicolumn{2}{|c|}{ Leaf texture }} & Pubescent. & 1 & 1 & 0 & 1 \\
\hline 33 & & & & Glabrous. & 0 & 0 & 1 & 0 \\
\hline 34 & & \multirow{4}{*}{\multicolumn{2}{|c|}{ Leaf length }} & $3-4 \mathrm{~cm}$. & 1 & 0 & 0 & 0 \\
\hline 35 & & & & $5 \mathrm{~cm}$. & 0 & 1 & 0 & 0 \\
\hline 36 & & & & $5-9 \mathrm{~cm}$. & 0 & 0 & 1 & 0 \\
\hline 37 & & & & $5-10 \mathrm{~cm}$. & 0 & 0 & 0 & 1 \\
\hline
\end{tabular}


Table (2): Cont.

\begin{tabular}{|c|c|c|c|c|c|c|c|c|}
\hline No & \multicolumn{4}{|c|}{ Character } & $\mathrm{A}$ & $\mathrm{B}$ & $\mathrm{C}$ & $\mathrm{D}$ \\
\hline 38 & \multirow{25}{*}{ 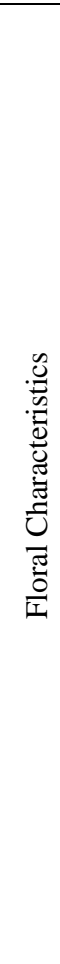 } & \multirow{2}{*}{\multicolumn{2}{|c|}{ Flower size }} & $4 \mathrm{~cm}$. & 1 & 1 & 0 & 0 \\
\hline 39 & & & & $5 \mathrm{~cm}$. & 0 & 0 & 1 & 1 \\
\hline 40 & & \multirow{4}{*}{\multicolumn{2}{|c|}{ Flower colour }} & Light pink. & 0 & 1 & 0 & 0 \\
\hline 41 & & & & White to pink. & 0 & 0 & 0 & 1 \\
\hline 42 & & & & Orange scarlet. & 1 & 0 & 0 & 0 \\
\hline 43 & & & & Red scarlet. & 0 & 0 & 1 & 0 \\
\hline 44 & & \multirow{5}{*}{$\begin{array}{l}\frac{n}{\tilde{a}} \\
\frac{\tilde{\partial}}{d}\end{array}$} & Number & 5 & 1 & 1 & 1 & 1 \\
\hline 45 & & & \multirow{4}{*}{ Shape. } & Erect. & 1 & 0 & 1 & 0 \\
\hline 46 & & & & Reflex. & 0 & 1 & 0 & 1 \\
\hline 47 & & & & Entire. & 1 & 0 & 1 & 1 \\
\hline 48 & & & & Serrulate. & 0 & 1 & 0 & 0 \\
\hline 49 & & \multirow{3}{*}{$\begin{array}{l}0 \\
\frac{\pi}{0} \\
2\end{array}$} & Number & 5 & 1 & 1 & 1 & 1 \\
\hline 50 & & & \multirow[t]{2}{*}{ Texture. } & Waxy. & 0 & 0 & 1 & 0 \\
\hline 51 & & & & Not waxy. & 1 & 0 & 0 & 0 \\
\hline 52 & & \multirow{2}{*}{\multicolumn{2}{|c|}{ Stamens number }} & More than 25 & 1 & 1 & 1 & 0 \\
\hline 53 & & & & $20-25$ & 0 & 0 & 0 & 1 \\
\hline 54 & & \multirow{5}{*}{$\begin{array}{l}\vec{E} \\
\overrightarrow{0}\end{array}$} & Type & Inferior. & 1 & 1 & 1 & 1 \\
\hline 55 & & & Carpels & 5 united. & 1 & 1 & 1 & 1 \\
\hline 56 & & & Placenta & Axile. & 1 & 1 & 1 & 1 \\
\hline 57 & & & Styles & Free. & 0 & 0 & 0 & 1 \\
\hline 58 & & & & Joined at base. & 1 & 1 & 1 & 0 \\
\hline 59 & & \multirow[b]{2}{*}{$\dot{0}^{\dot{0}}$} & Number & Many. & 1 & 1 & 1 & 1 \\
\hline 60 & & & type & Anatropous. & 1 & 1 & 1 & 1 \\
\hline 61 & & \multirow{2}{*}{\multicolumn{2}{|c|}{ Inflorescence. }} & Solitary. & 0 & 1 & 0 & 1 \\
\hline 62 & & & & In small cluster. & 1 & 0 & 1 & 0 \\
\hline 63 & \multirow{8}{*}{ 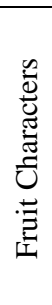 } & \multirow{4}{*}{\multicolumn{2}{|c|}{ Shape }} & Glubose. & 1 & 0 & 0 & 0 \\
\hline 64 & & & & Oblong. & 0 & 1 & 0 & 0 \\
\hline 65 & & & & Glubose to ovoid. & 0 & 0 & 1 & 0 \\
\hline 66 & & & & Pyriform. & 0 & 0 & 0 & 1 \\
\hline 67 & & & Type & Pomme. & 1 & 1 & 1 & 1 \\
\hline 68 & & \multirow{3}{*}{\multicolumn{2}{|c|}{ size }} & Large. & 0 & 1 & 0 & 1 \\
\hline 69 & & & & Medium. & 0 & 0 & 1 & 0 \\
\hline 70 & & & & Small. & 1 & 0 & 0 & 0 \\
\hline 71 & \multirow{6}{*}{ 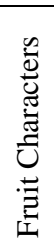 } & \multirow{3}{*}{\multicolumn{2}{|c|}{ colour }} & Yellow. & 0 & 1 & 0 & 1 \\
\hline 72 & & & & Yellow red. & 1 & 0 & 0 & 0 \\
\hline 73 & & & & Green yellow. & 0 & 0 & 1 & 0 \\
\hline 74 & & \multirow{3}{*}{\multicolumn{2}{|c|}{ Specific features }} & Fragrant. & 0 & 1 & 0 & 1 \\
\hline 75 & & & & Naked cav. & 0 & 1 & 0 & 0 \\
\hline 76 & & & & Calyx persist. & 0 & 0 & 0 & 1 \\
\hline
\end{tabular}


Table (2) Cont.

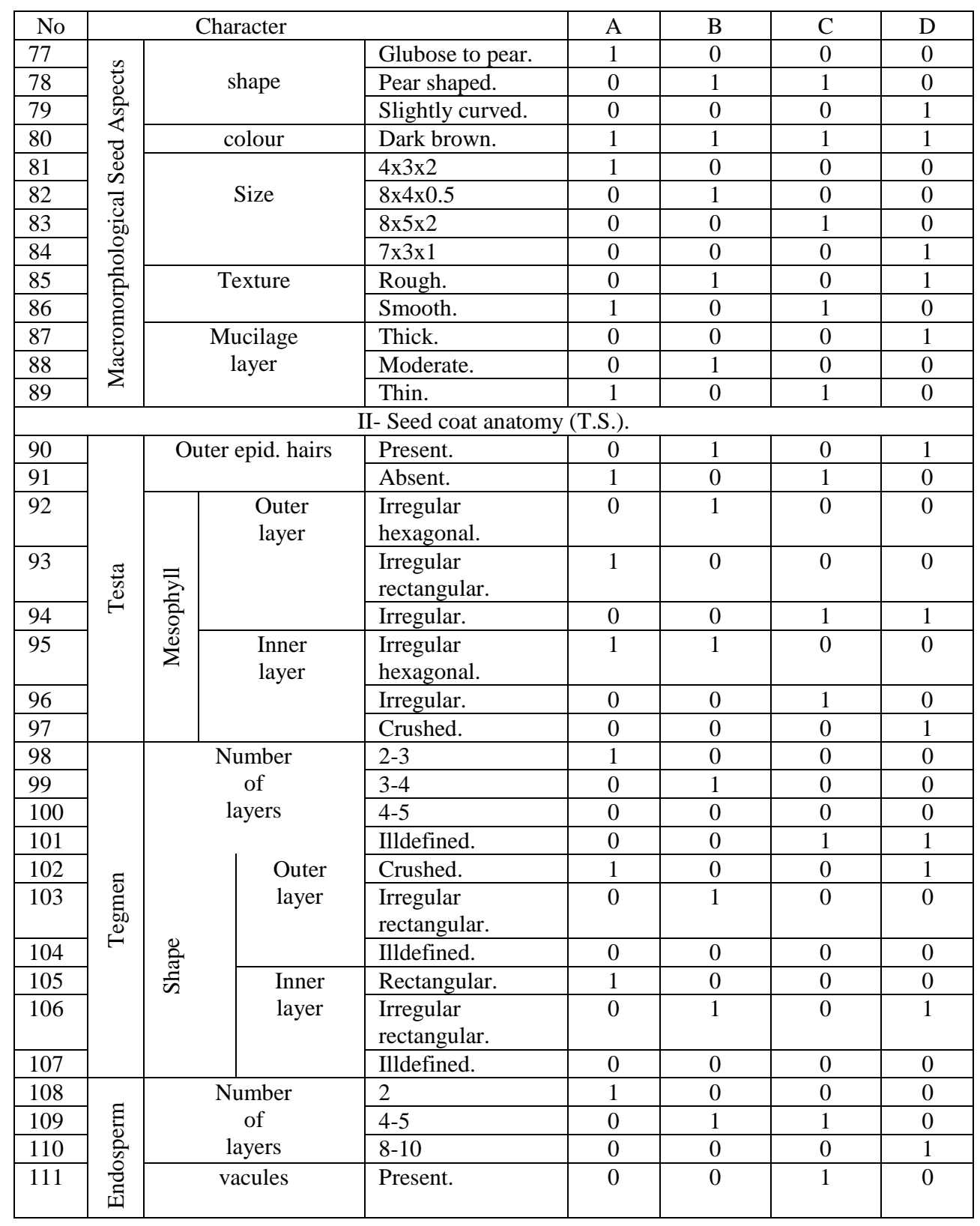


Table (2): Cont.

\begin{tabular}{|c|c|c|c|c|c|c|c|}
\hline \multicolumn{8}{|c|}{ III- Seed coat structure (SEM) } \\
\hline No & \multicolumn{3}{|c|}{ Characters } & $\mathrm{A}$ & $\mathrm{B}$ & $\mathrm{C}$ & $\mathrm{D}$ \\
\hline 112 & \multirow{3}{*}{\multicolumn{2}{|c|}{$\begin{array}{l}\text { Over all seed coat } \\
\text { pattern }\end{array}$}} & Reticulate to colliculate. & 1 & 1 & 0 & 0 \\
\hline 113 & & & Reticulate. & 0 & 0 & 1 & 0 \\
\hline 114 & & & Irregular reticulate. & 0 & 0 & 0 & 1 \\
\hline 115 & \multirow{5}{*}{ 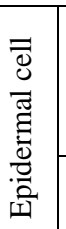 } & \multirow[t]{4}{*}{ Shape } & Irregular penta. to hexa. & 1 & 0 & 0 & 0 \\
\hline 116 & & & Rounded. & 0 & 1 & 0 & 0 \\
\hline 117 & & & Pentagonal to rounded. & 0 & 0 & 1 & 0 \\
\hline 118 & & & Irregular hexagonal. & 0 & 0 & 0 & 1 \\
\hline 119 & & Size & Monomorphic. & 1 & 1 & 1 & 1 \\
\hline 120 & \multirow{2}{*}{\multicolumn{2}{|c|}{ Hairs }} & Present. & 0 & 1 & 0 & 1 \\
\hline 121 & & & Absent. & 0 & 0 & 0 & 0 \\
\hline 122 & \multirow{15}{*}{ 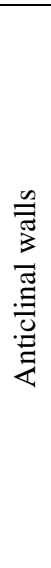 } & \multirow[t]{3}{*}{ Undulation } & Straight. & 1 & 0 & 0 & 0 \\
\hline 123 & & & Wavy. & 0 & 0 & 0 & 1 \\
\hline 124 & & & Curved. & 0 & 1 & 1 & 0 \\
\hline 125 & & \multirow[t]{4}{*}{ Thickness } & Very thin. & 0 & 1 & 0 & 0 \\
\hline 126 & & & Thin. & 1 & 0 & 0 & 0 \\
\hline 127 & & & Slightly thick. & 0 & 0 & 0 & 1 \\
\hline 128 & & & Very thick. & 0 & 0 & 1 & 0 \\
\hline 129 & & \multirow[t]{5}{*}{ Texture } & Rough. & 1 & 1 & 1 & 1 \\
\hline 130 & & & Smooth. & 0 & 0 & 0 & 0 \\
\hline 131 & & & Highly striated. & 1 & 1 & 0 & 0 \\
\hline 132 & & & Striated. & 0 & 0 & 0 & 1 \\
\hline 133 & & & Not striated. & 0 & 0 & 1 & 0 \\
\hline 134 & & \multirow[t]{3}{*}{ Hight } & Flat. & 1 & 1 & 0 & 0 \\
\hline 135 & & & Slightly raised. & 0 & 0 & 0 & 1 \\
\hline 136 & & & Highly raised. & 0 & 0 & 1 & 0 \\
\hline 137 & \multirow{7}{*}{ 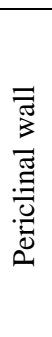 } & \multirow[t]{4}{*}{ Surface } & Flat. & 0 & 0 & 0 & 1 \\
\hline 138 & & & Flat to slightly concave. & 0 & 0 & 1 & 0 \\
\hline 139 & & & Slightly convex. & 1 & 0 & 0 & 0 \\
\hline 140 & & & Flat to slightly convex. & 0 & 1 & 0 & 0 \\
\hline 141 & & \multirow[t]{3}{*}{ Texture } & Highly striated. & 0 & 1 & 0 & 1 \\
\hline 142 & & & $\begin{array}{l}\text { Smooth with few } \\
\text { striations }\end{array}$ & 1 & 0 & 0 & 0 \\
\hline 143 & & & $\begin{array}{l}\text { Rough \& sligh. } \\
\text { tuberculate }\end{array}$ & 0 & 0 & 1 & 0 \\
\hline
\end{tabular}


Table (2): Continued

\begin{tabular}{|c|c|c|c|c|c|}
\hline & & \multicolumn{4}{|c|}{ IV-Seed protein electrophoresis } \\
\hline No. & Mol.wt. (KD) & A & $\mathrm{B}$ & $\mathrm{C}$ & D \\
\hline 144 & 103 & 0 & 0 & 1 & 0 \\
\hline 145 & 102 & 1 & 0 & 0 & 0 \\
\hline 146 & 97 & 0 & 0 & 0 & 1 \\
\hline 147 & 92 & 1 & 1 & 0 & 0 \\
\hline 148 & 90 & 1 & 0 & 1 & 1 \\
\hline 149 & 80 & 1 & 1 & 0 & 0 \\
\hline 150 & 69 & 1 & 0 & 1 & 1 \\
\hline 151 & 63 & 0 & 1 & 0 & 0 \\
\hline 152 & 61 & 1 & 0 & 1 & 0 \\
\hline 153 & 59 & 1 & 1 & 1 & 1 \\
\hline 154 & 52 & 1 & 1 & 1 & 1 \\
\hline 155 & 50 & 0 & 1 & 1 & 0 \\
\hline 156 & 48 & 0 & 0 & 1 & 0 \\
\hline 157 & 54 & 1 & 1 & 0 & 1 \\
\hline 158 & 42 & 1 & 1 & 1 & 0 \\
\hline 159 & 41 & 1 & 1 & 0 & 1 \\
\hline 160 & 40 & 1 & 0 & 0 & 0 \\
\hline 161 & 38 & 1 & 0 & 1 & 0 \\
\hline 162 & 36 & 0 & 1 & 0 & 0 \\
\hline 163 & 34 & 1 & 0 & 1 & 0 \\
\hline 164 & 32 & 0 & 1 & 0 & 1 \\
\hline 165 & 30 & 0 & 1 & 0 & 0 \\
\hline 166 & 28 & 1 & 0 & 0 & 0 \\
\hline 167 & 25 & 1 & 1 & 1 & 1 \\
\hline 168 & 21 & 1 & 0 & 0 & 0 \\
\hline 169 & 20 & 1 & 0 & 1 & 0 \\
\hline 170 & 18 & 0 & 1 & 0 & 0 \\
\hline 171 & 14 & 0 & 1 & 0 & 0 \\
\hline 172 & 11 & 0 & 0 & 0 & 1 \\
\hline 173 & 9 & 0 & 0 & 1 & 0 \\
\hline 174 & 8 & 1 & 1 & 1 & 0 \\
\hline 175 & 6 & 0 & 0 & 1 & 0 \\
\hline 176 & 5 & 0 & 1 & 0 & 0 \\
\hline 177 & 4 & 1 & 0 & 0 & 1 \\
\hline 178 & 3 & 0 & 0 & 1 & 1 \\
\hline Total & no. of bands & 20 & 17 & 17 & 12 \\
\hline
\end{tabular}




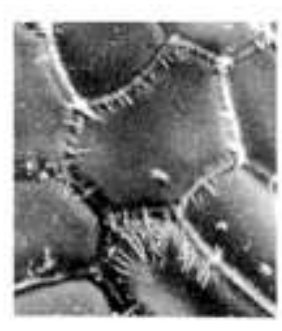

A

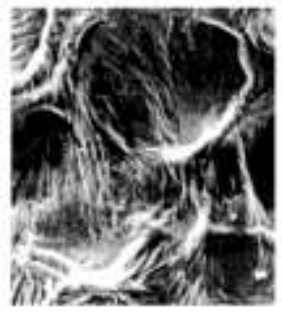

B

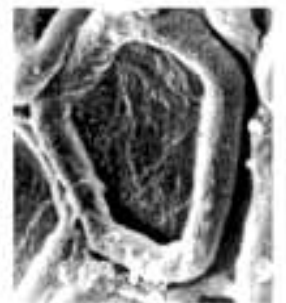

C

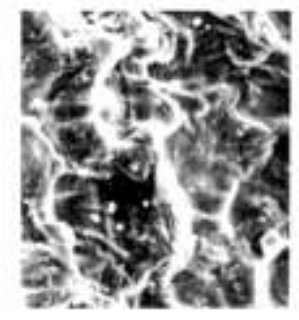

D

Fig. (1): Scanning electron micrographs of the studied taxa.

(A) at $\mathrm{x}=1500(\mathrm{~B} \& \mathrm{D})$ at $\mathrm{x}=1000(\mathrm{C})$ at $\mathrm{x}=2000$

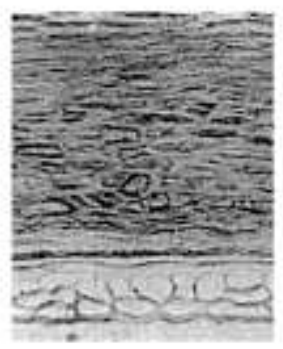

A

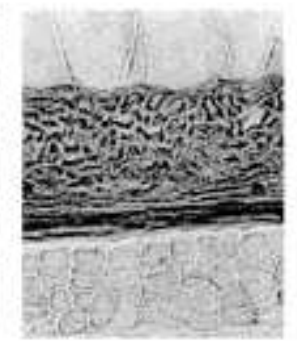

B

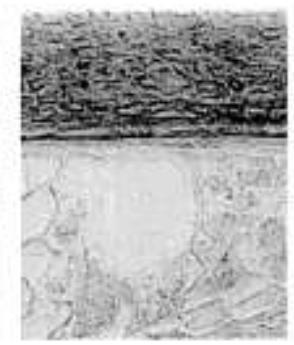

$\mathrm{C}$

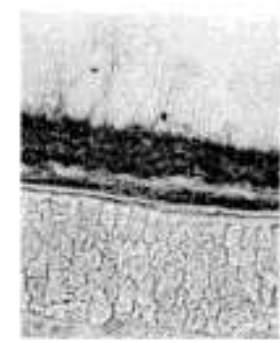

$\mathrm{D}$

Fig. (2): T.S. in seed coats of the studied taxa.

(A) at $\mathrm{x}=320(\mathrm{~B} \& \mathrm{D})$ at $\mathrm{x}=256(\mathrm{C})$ at $\mathrm{x}=200$

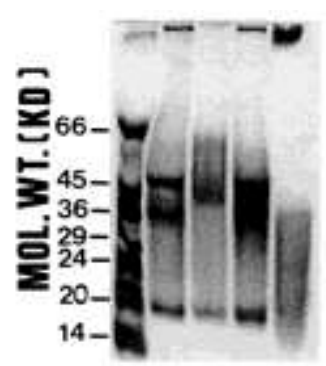

A

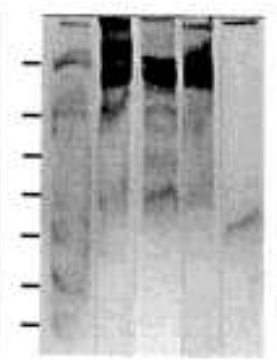

B

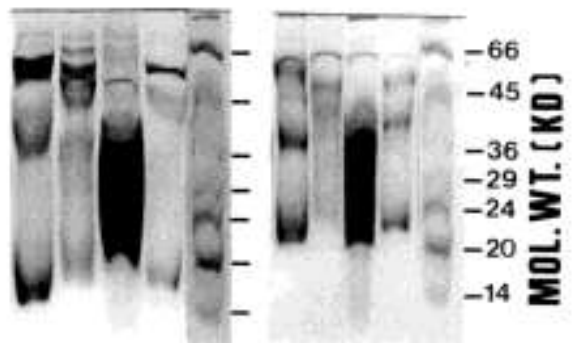

C
D

Fig. (3): Electrophoretic banding profiles of seed protein extracted in
(a) Tris- $\mathrm{HCl}$ (under reducing condition)
(b) Tris- $\mathrm{HCl}$ (under non-reducing condition)
(c) Tris-EDTA
(d) Tris-Glycine buffers of the studied taxa 
Average taxonomic distance (dissimilarity)

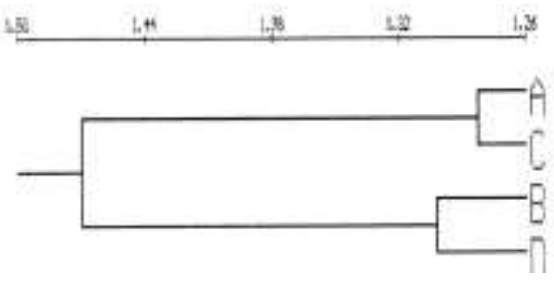

(a)

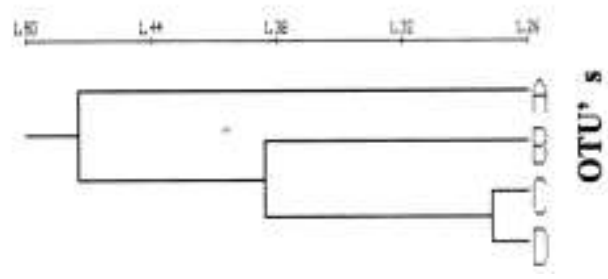

(b)

Average taxonomic distance (dissimilarity)

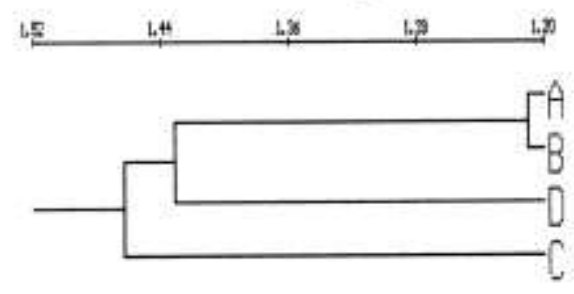

(c)

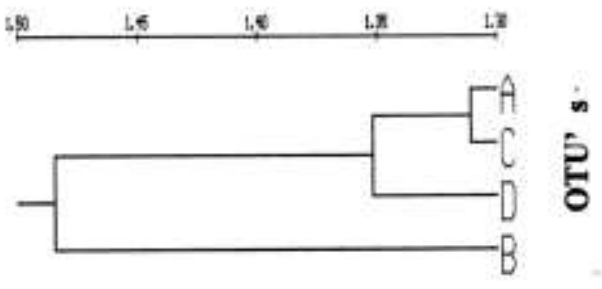

(d)

Average taxonomic distance (dissimilarity)

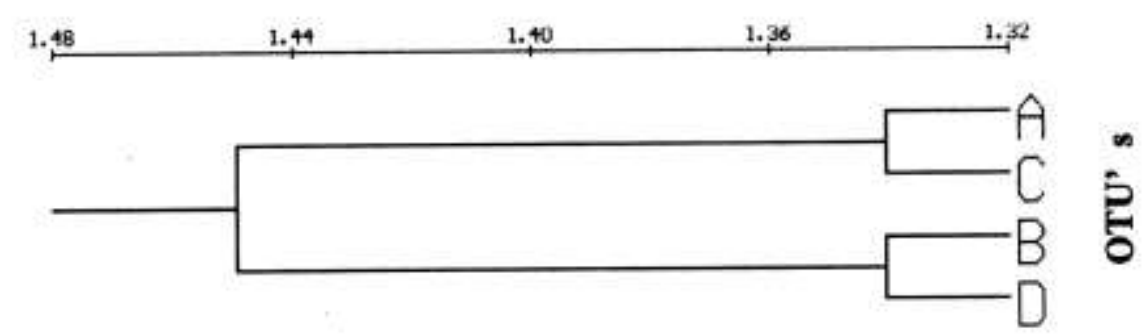

(e)

Fig. (4): UPGMA-phenogram based on:

(a) 89 morphological characters,

(b) 22 anatomical characters of seed coat T.S.,

(c) 32 attributes of seed coats scanning,

(d) 35 attributes obtained from SDS-PAGE profiles of seed proteins,

(e) 178 attributes (all characters), illustrating average taxonomic distance (dissimilarity) between the studied taxa. 
In the phenogram produced based on 22 anatomical characters of seed coats, Chaenomeles japonica is split off at 1.47, then Chaenomeles sinensis is split off at 1.38. Chaenomeles speciosa and Cydonia oblonga are grouped together and then divided at the dissimilarity level of 1.28 (Fig. 4-b).

In the phenogram produced based on 32 attributes of seed coats scanning Chaenomeles speciosa is split off at 1.47, then Cydonia oblonga is split at 1.43 average taxonomic distance. Chaenomeles japonica and Chaenomeles sinensis are grouped together and separated at 1.21 dissimilarity level (Fig. 4-c).

In the phenogram produced based on 35 attributes obtained from seed protein electrophoresis Chaenomeles sinensis is split off at the dissimilarity level of 1.48 and then Cydonia oblonga is split off at 1.38 level. The two species (Chaenomeles japonica and Chaenomeles speciosa) are grouped together and then separated from each other at 1.31 level of average taxonomic distance (Fig. 4-d).

The last phenogram produced based on all the studied characters clearly divided the four species into two groups (Fig. 4-e) at 1.45 level of dissimilarity, the first group included Chaenomeles japonica and Chaenomeles speciosa and the other one included Chaenomeles sinensis and Cydonia oblonga. The two species of the first group were separated from each other at 1.34 average taxonomic distance and at the same level the two species of the second group were separated from each other.

\section{Discussion}

The genera Chaenomeles Lindl. and Cydonia Miller have long been the subject of controversy among different authors (Table 3). Donoghue \& Sanderson (1992) have stressed on the importance of utilizing different criteria, both morphological and molecular, in reconstructing plant phylogeny and in the re-assessment of relationships between taxa. In their opinion, using only one or few criteria can be misleading.

In the present study, the phenogram constructed according to the analysis of 89 macromorphological characters showed a closer relation between Chaenomeles japonica and Chaenomeles speciosa on one hand and between Chaenomeles sinensis and Cydonia oblonga on the other hand. The former two taxa clustered at the dissimilarity level of 1.30 and the latter at 1.31. This result was in accordance with Bailey \& Bailey (1976) and Mabberley (1997) who transferred Chaenomeles sinensis to Cydonia to become Cydonia sinensis. Chaenomeles japonica and Chaenomeles speciosa were shown to be closely related by Rohrer et al. (1991), from studying the fruit structure, morphologically and anatomically.

In the phenogram constructed according to the analysis of 22 anatomical characters of seed coats, the results were generally inconclusive. Here, Chaenomeles japonica was split off from the other three taxa at a high dissimilarity level of 1.47 , while Chaenomeles speciosa was grouped with Cydonia oblonga at a low dissimilarity level of 1.28. This result was contradictory to the relevant literature that stated the close relation between Chaenomeles japonica and Chaenomeles speciosa as they readily hybridize among themselves (Mabberley, 1997). These results may be due to the analyzing of only 22 characters. It is worth mentioning that Corner (1976) stated that in the Maloideae in general, their seeds, offer no striking microscopic structure except their well developed testa, suggesting their close relationships and recent common ancestry. 
Table (3): A survey of the taxa recorded in Chaenomeles and Cydonia showing the controversies encountering their delimitation.

\begin{tabular}{|c|c|c|}
\hline $\begin{array}{c}\text { Taxon } \\
\text { no. }\end{array}$ & $2 n$ & Taxon status \\
\hline 1 & 34 & $\begin{array}{l}\text { Chaenomeles cathayensis (Hemsley) Schneid. } \\
\text { (Schneider, 1912; Bailey \& Bailey, 1976; Hillier, } 1981 \text { and Beckett 1983) } \\
\text { Chaenomeles lagenaria var. cathayensis Rehd. } \\
\text { (Rehder, } 1927 \text { and Bailey, 1949) } \\
\text { Cydonia cathayensis Hemsley } \\
\text { (Hemsley, 1873; Makins, } 1948 \text { and Bean, 1950) }\end{array}$ \\
\hline 2 & $\begin{array}{l}34 \\
34\end{array}$ & $\begin{array}{l}\text { Chaenomeles lagenaria Koidz. (Bailey, 1949) } \\
\text { =Chaenomeles speciosa (Sweet) Nakai } \\
\text { (Bailey \& Bailey, 1976; Hillier, } 1981 \text { and Mabberley, 1997) } \\
\text { Cydonia lagenaria Loisel } \\
\text { (Makins, } 1948 \text { and Bean, 1950) }\end{array}$ \\
\hline 3 & 34 & $\begin{array}{l}\text { Chaenomeles japonica Lindley } \\
\text { (Bailey, 1949; Bailey \& Bailey, 1976; Hillier, 1981; Beckett, } 1983 \text { and Mabberley, 1997) } \\
\text { Cydonia japonica Lindley. } \\
\text { (Makins, } 1948 \text { and Bean, 1950) }\end{array}$ \\
\hline 4 & 34 & $\begin{array}{l}\text { Chaenomeles sinensis Koehne } \\
\text { (Koehne, } 1893 \text { and Bailey, 1949) } \\
\text { Cydonia sinensis Thouin } \\
\text { (Makins, 1948; Bean, 1950; Bailey \& Bailey, } 1976 \text { and Mabberley, 1997) } \\
\text { Pseudocydonia sinensis Schneid. } \\
\text { (Schneider, 1912; Hillier, } 1981 \text { and Robertson } \text { et. al., 1992) }\end{array}$ \\
\hline 5 & 34 & $\begin{array}{l}\text { Cydonia oblonga Mill. } \\
\text { (Agreed about its status among different authorities) }\end{array}$ \\
\hline
\end{tabular}

$2 n=$ Basic chromosome number as reported in Fedorov 1969.

The phenogram constructed according to the analysis of 32 attributes of seed coat scan showed the grouping of Chaenomeles japonica and Chaenomeles sinensis at a 1.21 dissimilarity level. This suggested a close relation between the two taxa and so the retaining of Chaenomeles sinensis in the genus Chaenomeles as proposed by (Koehne, 1890; Bailey 1949 a and Mabberley, 1987). The splitting of Chaenomeles speciosa from the other three taxa at dissimilarity level of 1.47, then Cydonia oblonga at 1.43 followed by the other two taxa, gives an indication that generic limits cannot be drawn sharply between the two genera. A fact that was stated by Fernald (1947), Bailey (1949) and Aldasoro et al. (1998) for the Rosaceae in general and the Maloideae in particular.

The phenogram constructed according to the analysis of 35 attributes obtained from seed protein electrophoresis showed that Chaenomeles sinensis is split off from the remaining taxa at the high dissimilarity level of 1.48. This result is in accordance with 
M. H. A. Loutfy, A. A. A. El-Mashad \& E. A. Kamel

Bean 1950 and Robertson et al. (1992), who stated that this taxon differed from all the other genera of the Maloideae in possessing leaves with cylindrical gland tipped teeth. The latter author placed it in the monotypic genus Pseudocydonia, as was proposed earlier by Schneider (1912) and Hillier (1981). The two species Chaenomeles japonica and Chaenomeles speciosa clustered together at the dissimilarity level 1.31. This result is in agreement with Bailey (1949a), Bean (1950), Weber (1964), Bailey \& Bailey (1976), Hillier (1981), Beckett (1983) and Mabberley (1987 \&1997).

The last phenogram based on all characters studied, delimited the taxa in the two genera Chaenomeles Lindl. and Cydonia Miller as presented in Bailey \& Bailey (1976) and Mabberley (1997), giving further support to the merging of Chaenomeles sinensis with the genus Cydonia, as Cydonia sinensis Thouin and as presented earlier in Bean (1950).

\section{Acknowledgement}

The authors wish to express their deepest gratitude to Prof. Dr. Abdel Salam M. AlNowaihi, Professor of plant taxonomy and ex-head of Botany Department, Faculty of Science, Ain Shams University for revising the manuscript, his useful criticism and his continuous encouragement.

\section{References}

Aldasoro, J. J., Aedo, C. and Navarro, C. (1998): Pome Anatomy of Rosaceae Sub-fam. Maloideae, with special reference to Pyrus. Ann. Missouri. Bot. Gard. 85: 518-527.

Badr, A., Abou-El-Enain, M. M. and El-Shazly, H. H. (1998): Variation in seed protein electrophoretic pattern and species relationships in Sesbania. Proceedings of the $6^{\text {th }}$ Egyptian Conference of Plant Sciences, Cairo University (24-26 November, 1998). Vol. III: 493-501.

Bailey, L. H. (1949): Manual of Cultivated Plants. Macmillan Publishing Comp., New York. . (1949 b): The Pyrus-Malus puzzle. Gentes. Herb. 8: 40-43.

................ and Bailey, E. Z. (1976): "Hortus Third. A Concise Dictionary of Plants Cultivated in the U.S. \& Canada". "Revised by staff of the L.H. Bailey Hortorium". Macmillan Publ. Co. New York.

Barthlott, W. (1981): Epidermal and seed surface characters of plants: systematic applicability and some evolutionary aspects. Nord. J. Bot. 1: 345-355.

Bean, W. J. (1950): Trees \& Shrubs Hardy in the British Isles. Vol. 1: From A-E, 568-572. John Murray, London.

Beckett, K. A. (1983): The Concise Encyclopedia of Garden Plants. Orbis Ltd. London.

Bircher, W. (1960): Gardens of The Hesperides. Anglo-Egyption Bookshop Cairo, pp. 321.

Boesewinkel, F. D. \& Bouman, F. (1984): The seed structure. In "Embryology of Angiosperms" B. M. Johri (Ed.). Springer-Verlage, Berlin, Heidelberg, New York, Tokyo. 638-681.

Brisson, J.D. and Peterson, R.I. (1976): A critical review of the use of scanning electron microscopy in the study of the seed coat. Scan. Elect. Mic 2: 477-495.

Campbell, C. S., Donoghue, M. J., Baldwin, B. G. and Wojeiechowski, M. F. (1995): Phylogenetic relationships in Maloideae (Rosaceae): Evidence from sequences of the internal transcribed spacers of nuclear ribosomal DNA and its congruence with morphology. Amer. J. Bot. 82: 903-918. 
Chernoff, M., Plitmann, U. and Kislev, M. E. (1992): Seed characters and testa texture in species of the Vicieae, their taxonomic significance. Isr. J. Bot., 41: 167-186.

Corner, E. J. H. (1976): The Seeds of Dicotyledons. Vol. (1 \&2): Cambridge Univ. Press. (1992): The pachychalaza in dicotyledons: primitive or advanced. Bot. J. Linn. Soc. 108: 15-19.

Donoghue, M. J. and Sanderson, M. J. (1992): The Suitability of Molecular and Morphological Evidence in Reconstructing Plant Phylogeny. In "Molecular Systematics of Plants." Soltis, P.S., Soltis, D.E. and Doyle, J.J. (Eds.). Chapman \& Hall-New York.

Duke, J. A. (1961): Preliminary revision of the genus Drymaria. Ann. Missouri. Bot. Gard. 48: 173.

Eames, A. J. (1961): Morphology of the Angiosperms. McGraw-Hill Book Company. New York.

Fedorov, A. A. (1969) (Ed.): Chromosome Number of Flowering Plants. Leningrad.

Fernald, M. L. (1947): Minor transfers in Pyrus (reasons for uniting Malus, Sorbus and Aronia with Pyrus). Rhodora 49: 229-233.

Haborne, J. B. and Turner, B. L. (1984): Plant Chemo-systematics. Academic Press, New York. Hemsley, W. B. (1873): Handbook of Hardy Trees, Shrubs and Herbaceous Plants. London.

Heywood, V. H. (1993): Flowering Plants of the World. Andromeda, Oxford Ltd.

Hillier, H. G. (1981): Hilliers Manual of Trees and Shrubs (5 th Edition). Van Nostrand Reinhold Company. New York-London.

Jensen, U. (1984): Legumin-like and Vicilin-like storage proteins in Nigella damascena (Ranunculaceae) and six other dicotyledonous species. J. Plant Physiol. 115: 161-170.

Kalkman, C. (1988): The phylogeny of the Rosaceae. Bot. Jour. Linn. Soc. 98: 37-59.

Khushk, M. T. and Vaughan, J. G. (1986): Seed structure in relation to taxonomy of the Abutileae. Pak. J. Bot. 18 (10): 191-197.

Koehne, E. (1890): Die Gattungen der Pomaceen. R. Gaertners. Verlags Buchhandlung Hermann Heyfelder. Berlin.

Kumar, D., Rangaswamy, N. S. \& Dinesh, K., (1984): SEM studies on seed surface of wild and cultivated species of Vigna Savi. Proc. Ind. Acad. Pl. Sc., 93: 35-42.

Mabberley, D. J. (1987): The Plant Book: A Portable Dictionary of the Higher Plants. Cambridge University Press New York.

(1997): The Plant-Book, A Portable Dictionary of the Vascular Plants. Cambridge Univ. Press.

Makins, F. k. (1948): The Idntification of Trees \& Shrubs. J. M. Dent \& Sons LTD. London.

Martin, A. C. (1946): The comparative internal morphology of seeds. Am. Midl. Nat. 36: 513660.

Matta, N. K., Gatehouse, J. A. and Boulter, D. (1981): The structure of legumin of Vicia faba L. a reappraisal. J. Exp. Bot. 32: 183-197.

McLean, R. C. and Ivimey-Cook, W. K. (1956): Textbook of Theoretical Botany. Vol. II Page 1651. Longmans \& Green, London.

Netolitzky, F. (1926): Anatomie der Angiospermen Samen. (Cited from Corner E. J. H. 1976, The Seeds of Dicotyledons. Cambridge Univ. Press).

Paino, D., Urzo, M., Pedalino, M., Grillo, S., Rao, R., Tucci, M., Urzo-M.P., Ng-NQ \& Monti, L. M. (1990): Variability in major seed proteins in different Vigna species. Cowpea genetic resources, 1: 90-110 (Niger).

Pechoutre, F. (1902): Contribution a l'etude du developpement de l'ovule et de la graine des Rosacees. Annls Sci. nat. (Bot.) Ser. 8, 16, 1-158.

Phipps, J. B., Smith, P. G. and Rohrer, J. R. (1990): A checklist of the subfamily Maloideae (Rosaceae). Canad. Journ. Bot. 68: 2209-2269. 
Phipps, J. B., Robertson K. R., Rohrer, J. R. and Smith, P. G. (1991): Origins and evolution of the subfamily Maloideae (Rosaceae). Syst. Bot. 16: 376-394.

Rehder, A. (1927): Manual of Cultivated Trees and Shrubs. New York.

Rezk, M. R. (1980): Seed structure as a phylogenetic criterion. A case of Plantago seed. Egypt. J. Bot. 23: 51-62.

Rezk, M. R. (1987): Variation in seed coat microsculpture in five species of Plantago. Alex. Sci. Exch. 8 (3).

Robertson, K. R., Phipps, J. B., Rohrer, J. R. and Smith, P. G. (1991): A synopsis of genera in Maloideae (Rosaceae). Syst. Bot. 16: 376-394.

Phipps, J. B. and Rohrer, J. R. (1992): Summary of leaves in the genera of the Maloideae. Ann. Missouri. Bot. Gard. 79: 81-94.

Rohrer, J. R., Robertson, K. R. and Phipps, J. B. (1991): Variation in structure among fruits of Maloideae (Rosaceae). Amer. J. Bot. 78 (12): 1617-1635. , Robertson, K. R. and Phipps, J. B. (1994): Floral morphology of Maloideae (Rosaceae) and its systematic relevance. Amer. J. Bot. 81: 574-584.

Rohlf, F.J. (1989): NTSYS-pc, Numerical taxonomy and multivariate analysis system. New York. Exeter Publ., Ltd.

Rudenko, I. S. and Rotaru, G. I. (1988): Morphological and anatomical features of the seeds of hybrid forms of apple, pear and quince. Izvestiya-Akademii-Nauk-Moldavskoi-SSR. Bioloicheskie-I-Khimicheskie-Nauki. No. 5, 15-20.

Schmit, V., Debouck, S. G. and Baudoin, J. P. (1996): Biogeographical and molecular observations of Phaseolus glabellus (Fabaceae: Phaseolinae) and its taxonomic status. Taxon 45: 493-501.

Schneider, C. K. (1912): Illustriertes Handbuch de Laubholzkunde 2 vols. Cited from Bean W. J. (1950). Trees and Shrubs Hardy in the British Isles. John Murray. London.

Stant, M. T. (1973): The role of the scanning electron microscope in plant. Kew Bull., 28: 105115.

Stearn, W. T. (1966): Botanical Latin. Nelson \& Sons. London. Pages 506-507.

Stebbins, G. L. (1950): Variation and Evolution in Plants. Oxford Univ. Press. London.

Sterling 1966: Comparative morphology of the carpel in the Rosaceae. VII. Pomoideae: Chaenomeles, Cydonia, Docynia. Amer. J. Bot. 53: 225-231.

Weber, C. (1964): The genus Chaenomeles (Rosaceae). J. Arnold Arboretum. 45: 161-205.

Xuhan, X. and Van-Lammeren, A. A. M. (1994): The ultrastructure of seed coat development in Ranunculus sceleratus. Acta-Botanica-Neerlandica. 43 (1): 27-37. 